\title{
Animation-based Validation of a Formal Model of Dynamic Virtual Organisations
}

\author{
J. S. Fitzgerald, J. W. Bryans, D. Greathead, C. B. Jones and R. Payne \\ School of Computing Science, Newcastle University, UK \\ John.Fitzgerald@ncl.ac.uk
}

\begin{abstract}
We describe a study of the industrial use of animation in the analysis of a formal model of information flow in dynamic virtual organisations (VOs). A generic formal model of a VO structure composed of autonomous agents sharing information was developed using the Vienna Development Method (VDM). An exploratory environment was also developed in which the model was animated via an interpreter running an application-specific script developed with domain experts. A user interface encouraged interaction with the model without requiring exposure to the formalism. The use of the interface and model by domain experts was observed and recorded before debrief, allowing us to draw conclusions about the suitability of formal models for exploring, in an industrial setting, the design of policies governing VOs.
\end{abstract}

Keywords: Formal Methods, Virtual Organisations, Animation

\section{INTRODUCTION}

Advances in network technology, software and services are enabling organisations and individuals to form loosely-coupled temporary alliances in order to take advantage of a business opportunity or respond to an acute crisis. Such alliances are often called Virtual Organisations (VOs), virtual enterprises or dynamic coalitions (we will use these terms interchangeably). VOs are dynamic, evolving as the environment changes, as resources become available or unavailable and as members join or leave. In many applications, the ability to analyse system-level properties such as information flow, security and privacy is particularly significant. However, the architects of VOs currently lack a basis on which to evaluate the consequences of the design decisions that they make regarding coalition architecture and policies.

Formal methods offer a means of analysing the properties of potentially complex systems like dynamic VOs. In 2007, we had an opportunity to study this in collaboration with the UK Defence Science and Technology Laboratory (Dstl), who have an interest in tools that will assist policy evaluation and design for dynamic coalitions. It was natural for us to take a formal approach, but we had to bear in mind that the domain experts in Dstl, as with many potential industry users, had limited experience with formal methods. In particular, there is a need for "entry level" formal techniques that allow domain experts some of the benefits of modelling and analysis without requiring exposure to the full formalism. In the work reported here, we developed (relatively simple) formal models in conjunction with domain experts and, just as important, developed a purpose-built environment in which models could be explored by domain experts, allowing them to study the consequences of alternative VO policy decisions.

Although each virtual organisation is unique, many share some common features such as dynamically changing membership, mechanisms for information transfer and authorisation structures. Faced with the wide range of systems that qualify as VOs, we mapped out a space of VO architectures on the basis of "dimensions" including membership, information representation, provenance, time and trust [2]. Each dimension represents a choice, e.g. of a particular membership policy, information representation etc. A particular VO might be described in terms of the choices made in each dimension. We developed formal models representing specific architectural decisions within each dimension and validated the approach by showing how it could characterise a VO architecture designed for collaborative development in the chemical engineering industry [3]. In our study with Dstl, we sought to answer the following questions, among others: 
Can we provide formal models and tools that help in architecting VOs and their related policies?

Our previous work suggested that an executable model could be configured with particular decisions relating to the dimensions of interest. Scenarios could be played out in this instantiated model in order to analyse the consequences of (combinations of) policy decisions.

Are users' perceptions of information flow in VOs consistent with such a model? Human decision makers in VOs should have an accurate picture of the state of the real VO and hence the consequences of their decisions. User errors are one of the most common reasons for (socio-technical) system failure. This is recognised in the context of military information systems in [9] where it is argued "The rewards achieved through good training are best achieved at the Human-Machine Interface level". Furthermore, the advent of the UK Network Enabled Capability has raised many issues with regard to information management [8].

Our approach to these questions was constructive; we developed an exploratory environment that allows domain experts who are not familiar with formal modelling notations the opportunity to experiment with alternative policy decisions on models of VOs. We first describe the modelling approach used (Section 2), the exploratory environment (Section 3) and then outline the case study undertaken with industry users in which a VO model is evaluated against a scenario (Section 4). We present conclusions and suggest further work (Section 5).

\section{MODELS OF VIRTUAL ORGANISATIONS}

The models of VOs used in our work were developed using the Vienna Development Method (VDM). VDM is a model-oriented formalism with strong tool support and a record of application in industrial settings that are new to formal methods. A theme in VDM-based research has been that of lowering the entry barrier to the industrial use of formal methods without compromising the underlying formalism [5]. A major feature of this has been the provision of robust tools (VDMTools [7]) as well as the extension of the modelling language [4] to handle object-oriented structuring, concurrency [6], distribution and real-time [11]. For details, readers are referred to the texts and the VDM Portal (http://www.vdmportal.org). The VDM modelling languages have a very large executable subset, and VDMTools contains an efficient interpreter, allowing models to be evaluated directly by testing them on user-defined scenarios.

VDM models are usually based on descriptions of system state given in terms of variables each of which belongs to a type constructed from abstract base types and type constructors such as records, sets, sequences and finite mappings. Data Types may be restricted by invariants. For example, a very simple VO model might have two state variables describing indexed collections of coalitions and agents, defined as follows. Coalitions are modelled as finite sets of agents.

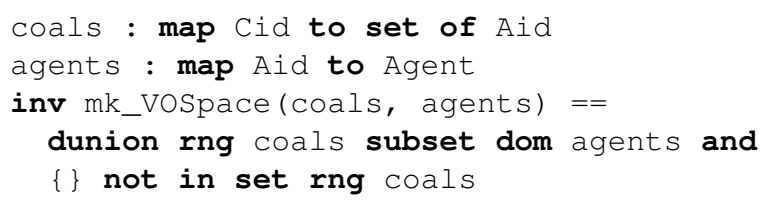

The invariant in this model is a predicate requiring that only known agents participate in coalitions and that no coalition is empty. The final conjunct embodies the constraint that no coalition (or virtual organisation) has a life independent of its members.

Functionality is described in VDM by operations specifying relations over the state variables, inputs and results. Operations may have side effects on state variables and may be defined with the aid of auxiliary functions. At the most abstract level, operations are specified in an implicit style by pre- and postconditions. For example, the following operation specification describes the removal of an agent from a coalition. The " " suffix on a state variable indicates the value in the variable before execution of the operation; postconditions are thus predicates of two states.

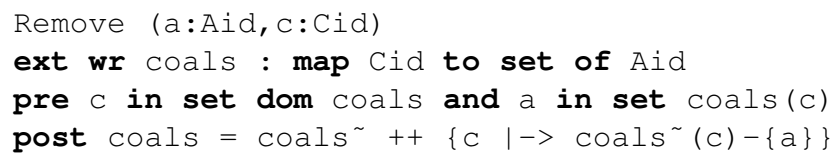


In VDM, proof obligations embody internal consistency constraints on models. For example, the satisfiability proof obligation requires that any operation specification should be capable of being realised by an implementation that preserves any invariants on the state variables. In the example above this condition would be broken: there is no constraint to ensure that the coalition $\mathrm{c}$ is not being emptied of its last member. This rigorous approach is one means by which questions are raised during the definition of a VO infrastructure, as we found with the chemical engineering example. In this case, on consulting with the engineers involved, it became clear that special procedures should be put in place to dissolve VOs, for example in order to ensure that shared information assets are not lost.

The model introduced above deals only with the membership aspect of a VO. Other models [2] have been developed for other aspects. For our study with Dstl, we used two VO models which both allow information to be stored at the level of the VO as well as within the participant agents. The two models differ in the operation that added members to the $\mathrm{VO}$, in each case offering a different policy regarding information transfer to new members.

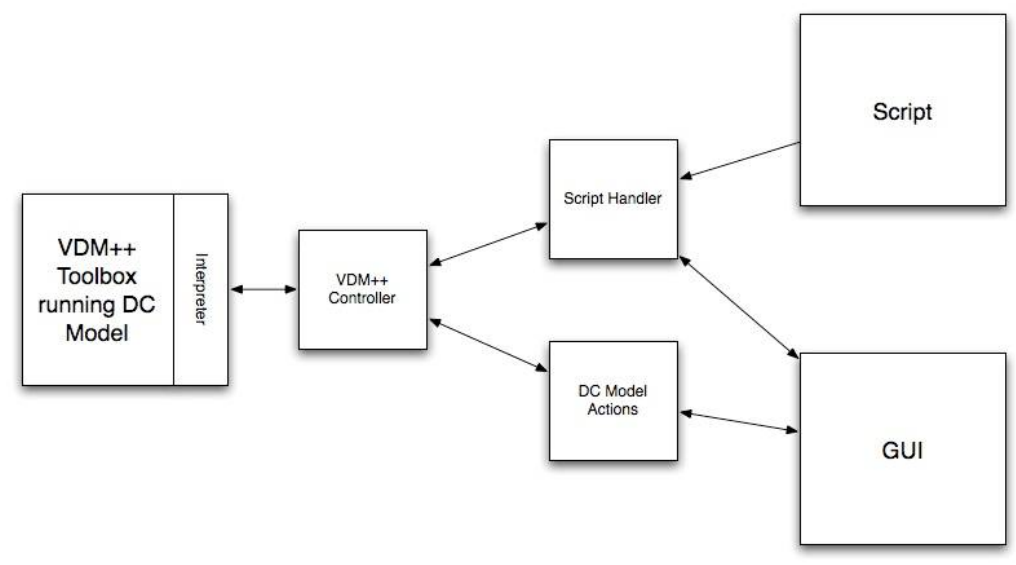

Figure 1: Outline architecture of the VO exploratory environment

\section{AN EXPLORATORY ENVIRONMENT}

The purpose of our exploratory environment was to provide a facility for domain experts to interact with a VO model without requiring familiarity with the notation. This is done primarily by running a scenario which invokes the operations in the VO model, running them on the VDMTools interpreter.

\subsection{Environment Architecture}

Figure 1 is a simplified view of the environment's architecture, based on a Model-View-Controller architecture. The VDM model contains information regarding the agents, the coalition structures and the information in the scenario. The view is provided by a Java graphical user interface (GUI) which allows the user to view the data in the model. The scenario script and Java controller (script handler) classes prompt and manage interaction from the user via the view, and pass any changes of state data to the model.

In order to support analysis by execution in the interpreter, directly executable versions of the operations are constructed in VDM. These retain the pre- and postconditions (which can be used as run-time checks). For example, the Remove operation above might be defined explicitly as follows, with a body containing an assignment to the state variable.

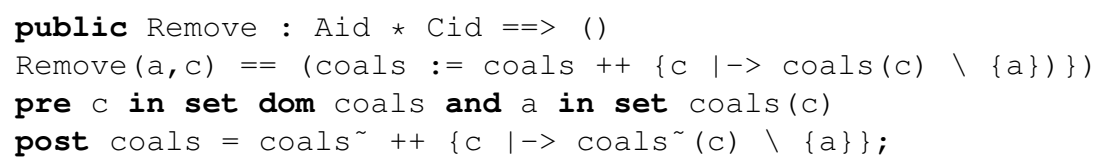

The scenario is encoded as a script in Java. This makes calls to the VDMTools application programmer interface through which commands are passed to the interpreter running the VO model. For example, the 
following Java source invokes operations to create a new agent in the VO model and add it to a VO called the "response coalition":

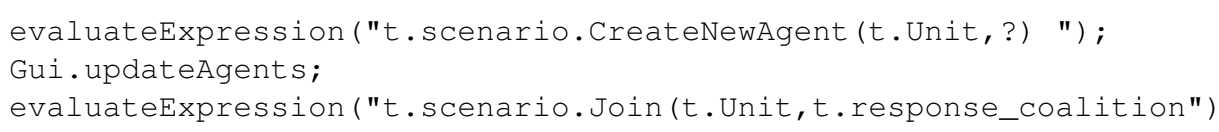

The execution of the Java causes the interpreter to execute the following explicitly-defined operations in the VO model:

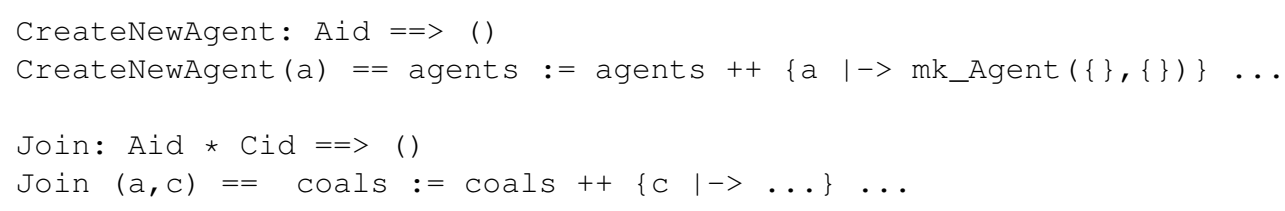

\subsection{User Interface and Scenario}

The GUI relays state data from the model to the user. It was developed using Java Swing and AWT libraries, giving us a platform-independent interface with the native look and feel of the operating system. The GUI consists of a tabbed pane containing the views onto the model (Fig. 2).

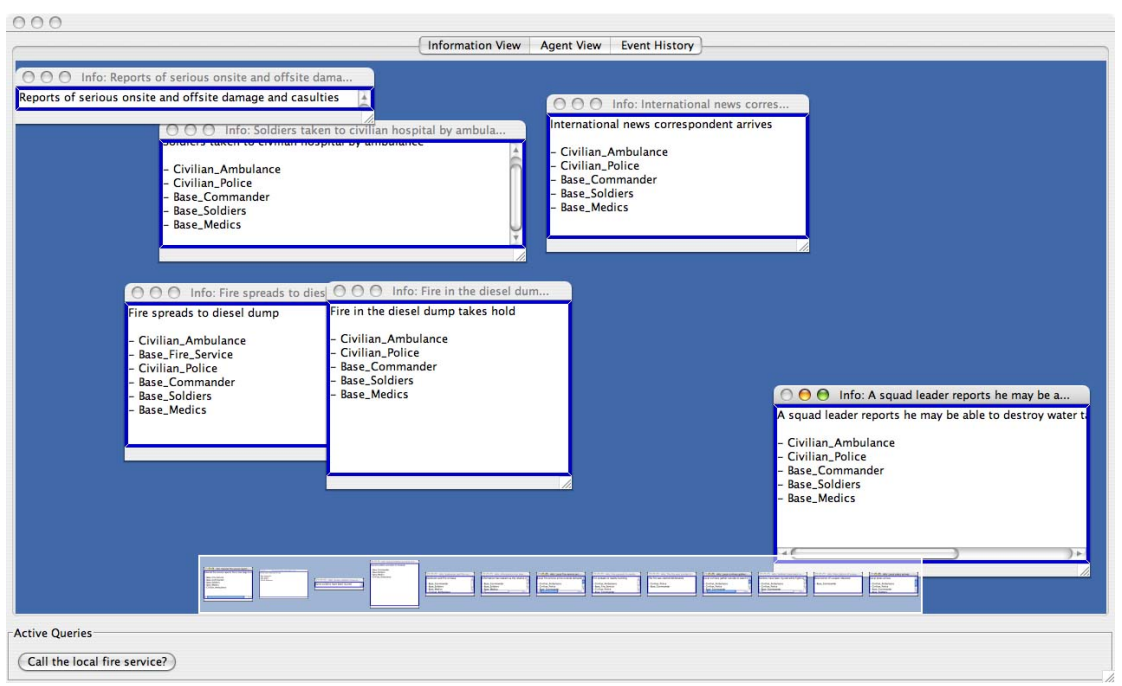

Figure 2: User interface to VO exploratory environment

The interface can show the model from two perspectives. The omniscient perspective reveals the full state of the model in terms of agents and the information that they contain. The participant perspective shows the point of view of a participating agent, showing only the information that has been the agent has derived for themselves or received from others. Each perspective may be examined from two main views. The information-oriented view provides one window for each piece of information presented to the user, indicating which agents are currently known to hold the information. The agent-oriented view shows each agent and the information items they hold. Coalition membership is represented using colour coding.

During operation the interface allows the user to manage information presented on the screen in the usual ways by moving, resizing, minimising and maximising windows. The arrangement is preserved when switching between views. In our study, the user took the participant perspective during execution of the scenario. After completion of the scenario, they were invited to use the omniscient perspective to see whether the distribution of information in the VO corresponded to their expectations.

The exploration environment that we developed was evaluated by means of a study in which the user is invited to make an initial policy choice regarding the disclosure of information to new VO members. The model corresponding to the relevant policy decision is initialised and animated on the interpreter using a scenario that was developed in conjunction with domain experts. 
The interface, driven by the script, displays items of information and questions (decision points) to the user. The user simply acknowledges new information as it is read but may respond to decision points by selecting from a range of alternatives offered by the script. Each item of information, and all decisions made by the user, are recorded in an event history log which is saved for reference after completion of the scenario.

The scenario was developed in consultation with a domain expert from Dstl. It involves the management of an acute crisis (a fire) and the news that the fire was caused maliciously. The user plays the role of the manager of the site in which the fire occurs. Decision points built into the scenario concern the opening of the site to emergency services, the management of staff and the press. The study is primarily concerned with managing the membership of a single emergency response VO and the flow of information between participants in that VO. The user makes their own decisions about the value of information presented to them.

The scenario script includes rudimentary calculations to model processes in the environment such as spread of the fire or numbers of casualties. It also contains the possibility of an unanticipated "leak" of information from within the VO to agents outside. After completion of the scenario from the participant perspective, the user was invited to explore the state from the omniscient perspective, note the information leak and perhaps re-run the scenario to see if any alternative decisions made might which avoid the leak.

\section{CASE STUDY}

A case study [10] uses an individual or a small number of participants who are closely observed. It tends to be exploratory in nature. The main purpose of our case study was to assess the exploratory environment, observe and record user behaviour, and seek to identify aspects worthy of further study.

In informal initial studies, several participants used the environment to work through the scenario, allowing us to evaluate and debug it. Finally, a more formal case study was conducted in which the participant was a domain expert with military command experience. He ran the scenario several times, his behaviour was observed throughout and he took part in informal exploratory interviews after each run. The interviews were conducted in a non-directive way, allowing the participant to give their own thoughts rather then being led to answer specific questions.

The need for an appropriate non-textual presentation of the context information (e.g. a map showing locations of fire units etc.) was noted. The user also wanted a less constrained palette of actions available to him during the execution of the scenario. We had refrained from providing this because we did not want to have to reveal too much about the structure of the formal model to the user. Future work will address this. Following the study, the user was very positive about the value of having a model that could be explored in this interactive way. Furthermore, the formality of the model was seen as a considerable benefit in opening the possibility of specialist, rigorous analysis.

\section{CONCLUSIONS}

Our study was directed at two questions. First, we asked whether we can provide formal models and tools that help in designing the architectures of VOs and their related policies. We have shown how it is possible to explore the consequences of alternative policy decisions in a way that is accessible to domain experts. Second, we asked whether users' perceptions of information flow in VOs would be consistent with a model. The case study has demonstrated that it is possible to explore the interaction between participants in a VO in order to assess the validity of models developed. One promising area of exploration is the coupling of access control policy models with those of VO membership [1], allowing participants to adjust the access rights granted to other VO members as the organisation and its goals evolve.

The scenario contains two interwoven elements: a model of the environment in the form of the state variables and the operations that update them, and the control flow governing the sequence of events and decisions in the scenario. These two aspects could be teased apart, with an external environment model coupled to that of the VO. Environment processes such as the spread of a fire are already described by well-tried, but rarely formal, specialist models that could be linked into our exploratory environment. Furthermore, it is possible to widen the scope of the exploratory environment to allow multiple users to take the roles of concurrent actors in the scenario. 
We have only begun to exploit the benefits that accrue from the formality of the model. In particular we have only animated the model and have not used any formal static analysis beyond generating proof obligations. As we indicated at the outset, our aim is to show that it is possible to gain benefit from early use of formal modelling. When formal models are developed after a proof-of-concept implementation, the basic implementation often becomes the basis for the product and the model is left lagging behind, before eventually being abandoned.

In future work, we aim to provide proof-based validation of system-level VO properties, noting that the key challenge here is eliciting and formalising these properties. In a domain as weakly defined as that of network-enabled virtual organisations, it is to be hoped that exploratory environments such as these will allow the use of formal models from very early stages when the key abstractions underpinning the expression of system-level properties are as yet uncertain.

Acknowledgements: We are grateful to Tom McCutcheon, Helen Phillips and Olwen Worthington of Dstl for the stimulus to examine dynamic virtual organisations, and to Dstl for supporting the project to develop the exploratory environment. This work also been supported by the EU FP7 integrated project DEPLOY (www. deploy-project.eu), the EPSRC Platform Grant on Trustworthy Ambient Systems and the Interdisciplinary Research Collaboration on Dependability (DIRC: www.dirc.org.uk).

\section{Bibliography}

[1] J. W. Bryans and J. S. Fitzgerald. Formal Engineering of XAML Access Control Policies in VDM++. In M. Butler, M. G. Hinchey, and M. M. Larrondo-Petrie, editors, Formal Methods and Software Engineering: Proc. 9th Intl. Conf. on Formal Engineering Methods, ICFEM 2007, volume 4789 of Lecture Notes in Computer Science, pages 37-56. Springer-Verlag, 2007.

[2] J. W. Bryans, J. S. Fitzgerald, C. B. Jones, and I. Mozolevsky. Dimensions of Dynamic Coalitions. Technical Report 963, Newcastle University, School of Computing Science, May 2006.

[3] J. W. Bryans, J. S. Fitzgerald, C. B. Jones, and I. Mozolevsky. Formal Modelling of Dynamic Coalitions, with an Application in Chemical Engineering. In T. Margaria, A. Philippou, and B. Steffen, editors, Proc. 2nd Intl. Symp. on Leveraging Applications of Formal Methods, Verification and Validation. IEEE, 2007. See Tech. Report 981, School of Computing Science, Newcastle University.

[4] J. S. Fitzgerald and P. G. Larsen. Modelling Systems - Practical Tools and Techniques in Software Development. Cambridge University Press, 1998.

[5] J. S. Fitzgerald and P. G. Larsen. Triumphs and Challenges for the Industrial Application of ModelOriented Formal Methods. In T. Margaria, A. Philippou, and B. Steffen, editors, Proc. 2nd Intl. Symp. on Leveraging Applications of Formal Methods, Verification and Validation. IEEE, 2007. See Technical Report 999, School of Computing Science, Newcastle University.

[6] J. S. Fitzgerald, P. G. Larsen, P. Mukherjee, N. Plat, and M. Verhoef. Validated Designs for Objectoriented Systems. Springer Verlag, London, 2005. ISBN 1-85233-881-4.

[7] J. S. Fitzgerald, P. G. Larsen, and S. Sahara. VDMTools: advances in support for formal modeling in VDM. Technical Report 1057, Newcastle University, School of Computing Science, November 2007. To appear in ACM SIGPLAN Notices 2008.

[8] P. Houghton. Potential System Vunerabilities of a Network Enabled Force. In Proc. Coalition Command and Control in The Networked Era, 2004.

[9] T. Nash. A Time to Refocus C4ISTAR Training. RUSI Defence Systems, 10(1):114-115, June 2007.

[10] C. Robson. Real world research : a resource for social scientists and practitioner-researchers. Blackwell , 2002.

[11] M. Verhoef, P. G. Larsen, and J. Hooman. Modeling and Validating Distributed Embedded Real-Time Systems with VDM++. In J. Misra, T. Nipkow, and E. Sekerinski, editors, FM 2006: Formal Methods, pages 147-162. Lecture Notes in Computer Science 4085, 2006. 